
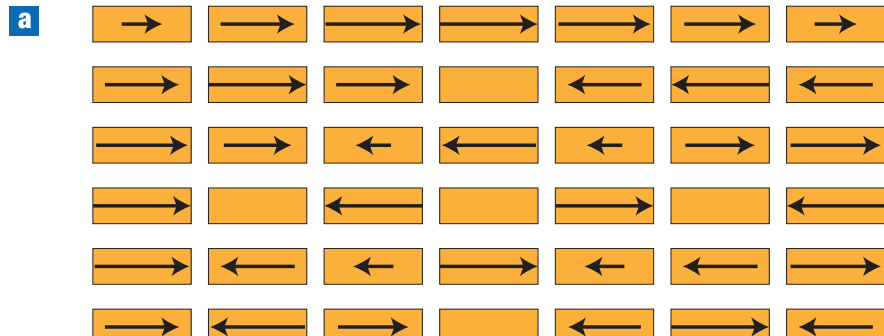

$\chi_{1} 540 \mathrm{THz} D^{\text {tot }}=2.51$

$\chi_{2} 560 \mathrm{THz} D^{\text {tot }}=0$

$\chi_{3} 588 \mathrm{THz} D^{\mathrm{tot}}=0.75$

$\chi_{4} 620 \mathrm{THz} D^{\text {tot }}=0$

$\chi_{5} 650 \mathrm{THz} D^{\mathrm{tot}}=0.33$

$\chi_{6} 675 \mathrm{THz} D^{\mathrm{tot}}=0$

$\chi_{7} 690 \mathrm{THz} D^{\mathrm{tot}}=0.10$

b
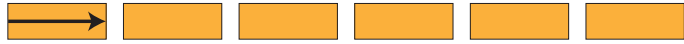

Figure 2 Nanorod chains. a, The frequencies, total dipole moments $\left(D^{\text {tot }}\right)$ and alignment of individual dipoles in the normal modes of a weakly coupled chain of 7 identical nanorods with dipolar resonances at $620 \mathrm{THz}$. b. An impulse excitation of the dipolar plasmon in the first nanorod of the chain can be viewed as a coherent superposition of all the eigenmodes of the systems.

In Fig. 2b, the excitation mechanism is illustrated. An impulse excitation at one end of the structure can be viewed as a coherent superposition of all of the individual eigenmodes. The excitation probability of each normal mode, $c_{i}$, is proportional to $1 / N$.

By exciting specific chain plasmon modes, Kawata et al. theoretically demonstrate that pixels of a subwavelength image can be transmitted efficiently from one side of a finite nanoparticle chain to the other. They also show that because the plasmon modes can form at several optical frequencies in the visible region, it should be possible for the approach to image pixels with specific colours. Further, by tapering the arrangement of the nanorod chains so that the ensemble is cone-shaped rather than cylindrical the subwavelength image can be magnified. This tapering is achieved by using a much larger spacing between chains at the image plane compared with the object plane (see Fig. 1).

The computational study by Kawata et al. clearly shows the possibility of accomplishing subwavelength colour imaging with low loss over far-field distances. The next challenge is experimental realization of the system.

\section{References}

1. Pendry, J. B. Phys. Rev. Lett. 85, 3966-3969 (2000).

2. Liu, N. et al. Nature Mater. 7, 31-37 (2008).

3. Kawata, S., Ono, A. \& Verma, P. Nature Photon. 2, 438-442 (2008).

4. Halas, N. J. Mater. Res. Soc. Bull. 30, 362-367 (2005).

5. Wang, H., Brandl, D. W., Nordlander, P. \& Halas, N. J. Acc. Chem. Res. 40, 53-62 (2007).

6. Maier, S. et al. Nature Mater. 2, 229-232 (2003).

\title{
PHOTONIC CIRCUITS
}

\section{Organic integration}

Integrated photonic circuits, such as lab-on-a-chip sensors, are expected to become increasingly important in the future. However, so far such photonic chips have typically been made from silicon and have lacked a vital ingredient - their own built-in light source. Although external sources can be used by coupling light into the chip through a lens or fibre, this significantly adds to the size, cost and complexity of the system, especially when several sources are required. The root of the problem lies with the difficulty in integrating lasers and LEDs made from compound semiconductors, such as GaAs, with a silicon platform.

It now appears that polymer photonic chips made from organic semiconductors could be a step closer to providing an answer to the problem. Malte Gather and co-workers from Universität zu Köln in Germany (Adv. Mater. 20, 1966-1971; 2008) have recently reported the ability to embed organic LEDs (OLEDs) into organic channel waveguides. The development suggests that it should be possible to create integrated all-organic photonic circuits that do not require an external light source.

Achieving an all-organic chip with integrated light sources is important because it could lead to mass-produced

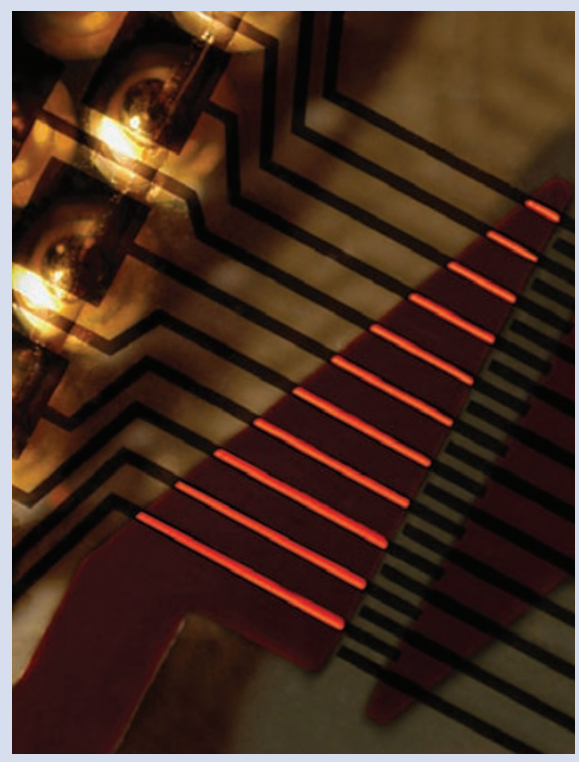

transparent matrix with a lower refractive index. The waveguide structure is made by spin-coating, and consists of a 500-nmthick hole-conducting bottom cladding, a 300-nm-thick electroluminescent waveguide core, which emits red light (wavelength $670 \mathrm{~nm}$ ), and a 300-nm-thick electron-conducting top cladding. Silver and copper layers are used as the cathode and anode electrodes, respectively, to electrically drive the light emission.

The resulting waveguide confines and guides the generated light and has a numerical aperture of 0.22 , corresponding to an acceptance and emission angle of $12.7^{\circ}$. To investigate the optical properties of the device, the team evaporate a triangular-shaped silver cathode layer on top of a set of parallel waveguides. Thus they create active regions - regions in electrical contact with the cathode and anode - with different lengths of miniature sensors that are cheap enough to become single-use disposable devices. The neat approach by Gather et al. is to use organic light-emitting material as the core of the channel waveguides used to transmit signals.

The device reported by Gather and co-workers is on the micrometre scale and features a multilayer waveguide buried in a the electrically excited waveguide. They observe that the normalized photocurrent increases as the length of the cathode layer increases, and that the average loss coefficient is $2.8 \pm 0.4 \mathrm{~dB} \mathrm{~mm}^{-1}$.

The next stage is to build complete organic photonic chips based on this principle.

Rachel Won 